\title{
Frequency of Erythrocyte Phenotypes in Blood Group Systems ABO and Rhesus at Moba, Province of Tanganyika, Democratic Republic of Congo
}

\author{
Bukasa Héman Kabemba ${ }^{1,2 *}$, Kabobo Ignace Kabobo ${ }^{1}$, Tchoni Savant Mukena ${ }^{1}$, \\ Mposhi Djolin Ngiele ${ }^{3}$, Nsomue Gentil Kabingie ${ }^{4}$, Tshinkobo Chicco Kasolva ${ }^{1}$, \\ Kabiswe Joëlle Pungwe1, Ebondo Patrick Kasendue ${ }^{3}$
}

\begin{abstract}
${ }^{1}$ Section of Nursing Sciences, Higher Institute of Medical Techniques of Kalemie, Kalemie, Democratic Republic of Congo ${ }^{2}$ Department of Public Health, Faculty of Health Sciences, University Institute of Congo, Moba, Democratic Republic of Congo ${ }^{3}$ Section of Nursing Sciences, Higher Institute of Medical Techniques of Lubao, Lubao, Democratic Republic of Congo ${ }^{4}$ Department of Internal Medicine, Faculty of Medicine, University of Kabinda, Kabinda, Democratic Republic of Congo Email: *hemanuska@gmail.com
\end{abstract}

How to cite this paper: Kabemba, B.H., Kabobo Wa Kabobo, I., Mukena, T.S., Ngiele, M.D., Kabingie, N.G., Kasolva, T.C., et al. (2017) Frequency of Erythrocyte Phenotypes in Blood Group Systems ABO and Rhesus at Moba, Province of Tanganyika, Democratic Republic of Congo. Open Access Library Journal, 4: e3421.

https://doi.org/10.4236/oalib.1103421

Received: February 3, 2017

Accepted: March 17, 2017

Published: March 20, 2017

Copyright $\odot 2017$ by authors and Open Access Library Inc.

This work is licensed under the Creative Commons Attribution International License (CC BY 4.0).

http://creativecommons.org/licenses/by/4.0/

\begin{abstract}
The determination of blood groups appears to be very useful in transfusion medicine, genetics, forensic medicine, organ transplantation and maternal alloimmunization. In Moba, blood transfusion is indicated in several anemias of the children. The present study aimed to determine the frequency of $\mathrm{ABO}$ and Rhesus blood groups in our environment. This is a descriptive cross-sectional study. Blood donors registered at the Moba General Reference Hospital for the period 2015 to 2016 were selected for this study $(n=2292)$. The Beth-Vincent test was used to determine blood group phenotypes from the monoclonal test sera. Blood groups $\mathrm{O}(60.5 \%)$ and $\mathrm{AB}(2.5 \%)$ were respectively the most frequent and the least encountered. In our series of studies, the numerical frequency order of the phenotypes of the $\mathrm{ABO}$ and Rhesus (D) blood groups included in order of importance: $\mathrm{O}^{+}(\mathrm{n}=1364$ or $59.5 \%) ; \mathrm{A}^{+}(\mathrm{n}=488$ or $21.3 \%)$; $\mathrm{B}^{+}(\mathrm{n}=348$ or $15.2 \%) ; \mathrm{AB}^{+}(\mathrm{n}=55$ or $2.4 \%) ; \mathrm{O}^{-}(\mathrm{n}=22$ or $1.0 \%) ; \mathrm{A}^{-}(\mathrm{n}=7$ or $0.3 \%) ; \mathrm{B}^{-}(\mathrm{n}=5$ or $0.2 \%)$; and $\mathrm{AB}^{-}(\mathrm{n}=3$ or $0.1 \%)$. This observed difference between the different $A B O$ and Rhesus groups is significant. Sex does not significantly affect the type of blood group. The distribution of $\mathrm{ABO}$ and rhesus groups follows the same Negroid distribution as in many countries. The results should be capitalized for proper priority management of the blood to be stored in the blood bank.
\end{abstract}

\section{Subject Areas}

Nursing, Public Health 


\section{Keywords}

ABO, Blood Group, Moba, Erythrocyte, Phenotype, Rhesus

\section{Introduction}

Le sang, tissu conjonctif liquide en mouvement composé des éléments figurés et du plasma dont le rôle vital a été connu dès la préhistoire, est un facteur important de vie [1]. Si la découverte de sa circulation s'était passé dès 1628 avec William Harvey, il a fallu attendre la découverte des groupes sanguins (à partir des années 1900, Karl Landsteiner) dont les connaissances ont permis le développement extraordinaire de la transfusion sanguine [1]-[6]. Le groupe sanguin est une classification du sang reposant sur la présence ou l'absence des antigènes hérités à la surface des cellules hématopoïétiques ou dans le plasma [7] [8] [9] [10]. Il existe plus de 600 antigènes et plusieurs systèmes de groupes sanguins évalués à environ 339 et repartis en 33 systèmes dont les $\mathrm{ABO}$ et Rhésus (Rh) qui sont des systèmes érythrocytaires qui font l'objet notre étude [3] [4] [5] [6] [11]. Ces antigènes (Ag) sont pour la plupart regroupés en systèmes génétiquement induits dont la connaissance est nécessaire dans plusieurs domaines: transfusion, allo-immunisation foeto-maternelle, transplantation des organes, études génétiques et médecine légale [4] [5] [6] [11] [12]. Le système de groupes sanguins $A B O$ est le principal type des groupes sanguins classés en quatre phénotypes (A, B, O et $\mathrm{AB}$ ) [4] [5] [6] [11] [12] selon la présence ou l'absence des antigène et anticorps spécifiques sur la membrane des hématies ou dans le plasma [7] [9] [10]. Ces antigènes (localisés sur le chromosome 9) sont de nature glucidique: $\mathrm{D}$-galactose pour l'antigène $\mathrm{B}$ et $\mathrm{N}$-acétylgalactosamine pour l'antigène A [1] [10] [13] [14]. Génétiquement le A et B sont des allèles Codominant et le $\mathrm{O}$ est récessif [13].

Le système rhésus est le second système de groupes sanguins érythrocytaires rencontré dans l'espèce humaine [15]. Il existe plusieurs antigènes protéiques (localisés sur le chromosome 1) dans ce système dont le principal est l'antigène $\mathrm{D}$, incriminé dans les réactions immunes [10] [15].

La transfusion sanguine est une thérapeutique dangereuse. Elle peut être à la base des accidents à risque mortel suite aux polymorphismes antigéniques d'origine génétique des individus et doit assurer pour se faire la sécurité du receveur pour deux missions: la compatibilité immunologique et l'absence de transmissions des maladies [1] [16]. La compatibilité immunologique est exigée entre le receveur et donneur du sang: iso-groupe et iso-rhésus [5] [7] [13].

La seule façon d'améliorer la sécurité immunologique des transfusés, surtout dans les pays en voie de développement, est le phénotypage des donneurs et receveurs des produits sanguins (sang total, plasma, culot globulaire, sang déleucocyté, etc.) par les groupages sanguins érythrocytaires, essentiellement $\mathrm{ABO}$ et Rhésus [1] [7]. Ce geste pourrait permettre l'administration du sang compatible aux patients qui en auront besoin [1] [16]. Il sied de noter qu'une 
incompatibilité sanguine peut entrainer le décès.

Plusieurs études existent au sujet des groupes sanguins [1]-[7]. Les auteurs évoquent à l'unanimité la prédominance du groupe sanguin $\mathrm{O}$ et de Rhésus positifs dans le monde [17], même si de façon purement isolé on peut rencontrer la prédominance soit du groupe A [17] [18] [19], soit du groupe O [11]-[16] [19]-[25] dans certains coins de la planète. Les individus porteurs de rhésus sont les plus rencontrés dans l'espèce humaine [7] [16]-[24]. En République Démocratique du Congo, particulièrement à Moba, les transfusions sanguines constituent une pratique courante, surtout en pédiatrie pour anémies décompensées. Aucune transfusion ne peut être faite sans détermination préalable des Groupes sanguins [16] [26]. Il nous a paru opportun d'entreprendre cette étude dans l'objectif de déterminer la fréquence de phénotypes des groupes sanguins $A B O$ et Rhésus et ainsi renforcer la gestion du sang à transfuser dans notre milieu.

\section{Matériels et Méthodes}

\subsection{Milieu, Type et Période D’étude}

Il s'agit d'une étude descriptive transversale. Les donneurs de sang enregistrés à l'hôpital général de référence de Moba (commune de Kirungu, ville de Kaoze dans Territoire de Moba) sur la période allant de 2015 à 2016 ont été retenus pour cette étude. (Figure 1)

Le territoire de Moba est situé au Sud-Est de la province du Tanganyika en République Démocratique du Congo (RDC). Il a une superficie de $24.500 \mathrm{Km}^{2}$ et la population estimée en 2015 à 609.406 habitants. Du point de vue ethno-tribal, on y rencontre principalement les Tabwa (plus de 80\%), Luba et Bemba [27].

\subsection{Population D'étude}

Nous avons recruté systématiquement tous les donneurs de sang sur qui le groupage sanguin ( $\mathrm{ABO}$ et $\mathrm{RH})$ a été effectué en lien avec nos critères d'inclusion. De ce fait, 2.292 personnes âgées de 15 à 67 ans avaient été retenues. L'accord des autorités a été obtenu avant d'entreprendre nos recherches. Pour être inclus, le donneur de sang devrait remplir les paramètres recherchés sur la période et lieu concerné par cette étude (HGR de Moba de 2015 à 2016).

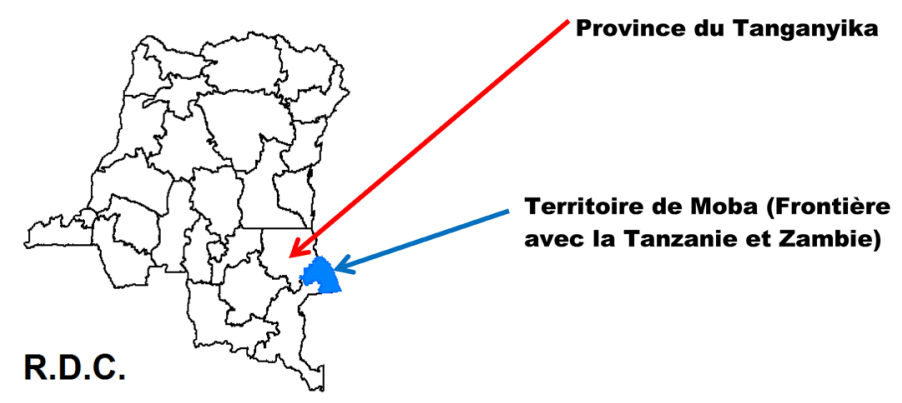

Figure 1. Position géographique du territoire de Moba en RDC Geographic localization of Moba in DRC. Source: Carte tirée de www.caid.cd [27]. 
Les paramètres d'étude comprenaient l'âge, le sexe et le type de groupe sanguin érythrocytaire ( $A B O$ et Rhésus). Le groupe sanguin (GS) $A B O$ avait quatre possibilités phénotypiques: $\mathrm{A}, \mathrm{B}, \mathrm{O}$ et $\mathrm{AB}$; alors que pour le Rhésus $\mathrm{D}$, le donneur a été classé Rhésus ( $\mathrm{RH}$ ou $\mathrm{Rh}$ ) positif ou négatif selon les résultats d'agglutination in vitro $(+=$ présence de l'agglutination).

\subsection{Détermination de Groupe Sanguin ABO et RH au Laboratoire}

Le groupe sanguin $\mathrm{ABO}$ et Rhésus ont été déterminés selon les principes immunologiques établis. Le groupage sanguin $\mathrm{Rh}$ est la recherche à la surface des hématies testées des allo- $\mathrm{Ag}$ courants du système Rhésus (l'Ag D, l'Ag C, l'Ag E, l'Ag $\mathrm{c}$ et l'Ag e) en mettant en contact ces hématies avec des Anticorps spécifiques connus: anti-D, anti-C, anti-c, anti-E et anti-e. Pour cette étude, c'est le sérum Anti-D qui avait été utilisé. Pour le $\mathrm{ABO}$, il existe deux méthodes pour déterminer les groupes sanguins sur plaque: Beth-Vincent et de SimoninMichon.Le laboratoire de l'HGR de Moba recours à la technique de BethVincent [28] ou épreuve globulaire qui consiste mettre en évidence les antigènes globulaires (hématies) en utilisant des sérums tests spécifiques connus anti - A, $\mathrm{B}$ et $\mathrm{AB}$.

Nous avons recherchés les différents phénotypes en procédant de la manière suivante: (i) Prélever 3 à 5 millilitre de sang sur tube sec ou avec anticoagulant (EDTA); (ii) Déposer dans l'ordre trois gouttes de sérum test (anti-A, anti-B et anti-D); (iii) Ajouter à côté de chaque goutte de sérum test, une goutte de culot d'hématies à tester (une petite goutte ou des hématies diluées).

Il est important qu'il n'y ait pas trop d'hématies, risquant d'empêcher l'observation correcte des agglutinations. (i) Mélanger soigneusement les deux gouttes puis animer la plaque d'un mouvement rotatoire lent; (ii) Observer au bout d'une minute et rechercher s'il y a ou non agglutination. On parle d'hémolyse in vivo et agglutination in vitro; (iii) La présence d'une agglutination fait noter (+) et son absence (-).

\subsection{Analyse Statistique des Donnees}

Pour rendre les données de notre étude présentable, nous avons eu recours aux logiciels Word et Excel (Microsoft, USA, 2010). Les calculs de la fréquence et de la moyenne avaient été mis à contribution dans l'expression des résultats. La différence entre les variables a été appréciée à l'aide du test de Khi-Carré non corrigé de Pearson au seuil significatif $\mathrm{p} \leq 0.05$ (erreur alpha).

\section{Resultats}

Les résultats de cette étude concernent les donneurs de sang du laboratoire biomédical de l'hôpital généralde référence de Moba. Deux milles deux-cents nonante-deux $(n=2292)$ résultats de groupage sanguin des donneurs de sang ont été analysés.

L'âge moyen des donneurs était de $37.6 \pm 1.8$ ans avec des extrêmes allant de 15 à 67 ans (Table 1). Les donneurs du sang de sexe féminin ont été plus 
représentés avec 50.4\% $(\mathrm{n}=1155)$ sans différence statistiquement significative $(\mathrm{p}>0.05)$.

Les résultats obtenus dans le Table 2 révèlent que les phénotypes du système de groupes sanguins $A B O$ ont été respectivement: $60.5 \%(n=1386)$ pour le $O$; $21.6 \%(\mathrm{n}=495)$ pour le $\mathrm{A} ; 15.4 \%(\mathrm{n}=353)$ pour le $\mathrm{B}$; et $2.5 \%(\mathrm{n}=58)$ pour le $A B$. La prédominance du groupe $O$ a été statistiquement significative $(\mathrm{p}<0.05)$ alors que le $\mathrm{AB}$ est le moins rencontré.

Les individus de sexe féminin ont été plus représentés dans notre échantillon avec 50.4\% $(\mathrm{n}=1.155)$. Les différences entre les sexes en faveur de la distribution des groupes sanguins $A B O$ n'était pas significative $(\mathrm{p}>0.05)$ (Table 2), même si les groupes $B(n=197)$ et $O(n=694)$ ont été les plus rencontrés chez les hommes et $A(n=268)$ et $A B(n=39)$ pour les femmes (Table 2).

Dans le système de groupes sanguins rhésus, les $\mathrm{Rh}$ (D) positifs ont été statistiquement les plus représentés $(\mathrm{p}<0.05)$, soit 98.4\% $(\mathrm{n}=2255)$. La proportion des rhésus (D) négatifs a été de trente-sept cas, soit 1.6\% (Table 3 et Table 4). Le sexe n'influe pas sur la survenue de rhésus positifs (différence non significative).

Dans notre série d'étude, l'ordre numérique de fréquence des phénotypes des

Table 1. Distribution des donneurs de sang par rapport à l'âge et sexe Distribution of blood donors groups: Age and sex.

\begin{tabular}{|c|c|c|c|c|}
\hline \multirow{2}{*}{ Age (ans) } & \multicolumn{2}{|c|}{ Sexe } & \multirow{2}{*}{ Total n (\%) } & \multirow{2}{*}{$\mathrm{p}$} \\
\hline & Masculin n (\%) & Féminin $\mathrm{n}(\%)$ & & \\
\hline [15 - 24[ & $220(9.6)$ & $227(9.9)$ & 447 (19.5) & \\
\hline [24 - 33[ & $339(14.8)$ & $422(18.4)$ & $761(33.2)$ & \\
\hline$[33-42[$ & $312(13.6)$ & $284(12.4)$ & $596(26.0)$ & $\mathrm{NS}^{*}$ \\
\hline$[42-51[$ & $188(8.2)$ & $112(4.9)$ & $300(13.1)$ & \\
\hline$[51-60[$ & $59(2.6)$ & $69(3.0)$ & $128(5.6)$ & \\
\hline$[60-69[$ & $19(0.8)$ & $41(1.7)$ & $60(2.6)$ & \\
\hline Total & $1137(49.6)$ & $1155(50.4)$ & $2292(100)$ & $\mathrm{NS}^{*}$ \\
\hline
\end{tabular}

Moyenne: 37.6 et Sd: 1.8 ; NS*: Non significatif; Sd: déviation standard.

Table 2. Distribution des GS du système ABO (ISBT 001) par rapport au sexe Distribution of Abo blood groups and sex.

\begin{tabular}{ccccc}
\hline \multirow{2}{*}{ GS } & \multicolumn{2}{c}{ SEXE } & \multirow{2}{*}{ TOTAL n (\%) } & p \\
\cline { 2 - 3 } & Masculin n (\%) & Féminin n (\%) & \\
\hline A & $227(9.9)$ & $268(11.7)$ & $495(21.6)$ & \\
B & $197(8.6)$ & $156(6.8)$ & $353(15.4)$ & $<0.05\left(\mathrm{~S}^{*}\right)$ \\
AB & $19(0.8)$ & $39(1.7)$ & $58(2.5)$ & \\
0 & $694(30.3)$ & $692(30.2)$ & $1386(60.5)$ & \\
Total n (\%) & $1137(49.6)$ & $1155(50.4)$ & $2292(100)$ & NS \\
\hline
\end{tabular}

$S^{*}$ : Significatif; ISBT: International Society of Blood Transfusion. 
Table 3. Distribution des GS rhésus (ISBT 0044) par rapport au sexe Distribution of rhesus blood groups and sex.

\begin{tabular}{|c|c|c|c|c|}
\hline \multirow{2}{*}{ SEXE } & \multicolumn{2}{|c|}{ Rhésus } & \multirow{2}{*}{ Total n (\%) } & \multirow{2}{*}{$\mathbf{P}$} \\
\hline & Positif n (\%) & Négatif n (\%) & & \\
\hline Masculin & $1123(49.0)$ & $14(0.6)$ & $1137(40.9)$ & NS \\
\hline Féminin & $1132(49.4)$ & $23(1.0)$ & $1155(50.4)$ & \\
\hline Total & $2255(98.4)$ & $37(1.6)$ & $2292(100)$ & $S$ \\
\hline
\end{tabular}

Table 4. Distribution des groupes sanguins ABO par rapport au Rhésus Distribution of $A B O$ and rhesus blood groups.

\begin{tabular}{ccccc}
\hline \multirow{2}{*}{ GS ABO } & \multicolumn{2}{c}{ Groupes sanguins Rhésus } & Total n (\%) & p \\
\cline { 2 - 3 } & $\mathrm{Rh}(+) \mathbf{n}(\%)$ & $\mathrm{Rh}(-) \mathbf{n}(\%)$ & & \\
\hline A & $488(21.3)$ & $7(0.3)$ & $495(21.6)$ & \\
B & $348(15.2)$ & $5(0.2)$ & $353(15.4)$ & $\mathrm{S}$ \\
0 & $1364(59.5)$ & $22(1.0)$ & $1386(60.5)$ & \\
AB & $55(2.4)$ & $3(0.1)$ & $58(2.5)$ & \\
Total & $2255(98.4)$ & $37(1.6)$ & $2292(100)$ & $\mathrm{S}$ \\
\hline
\end{tabular}

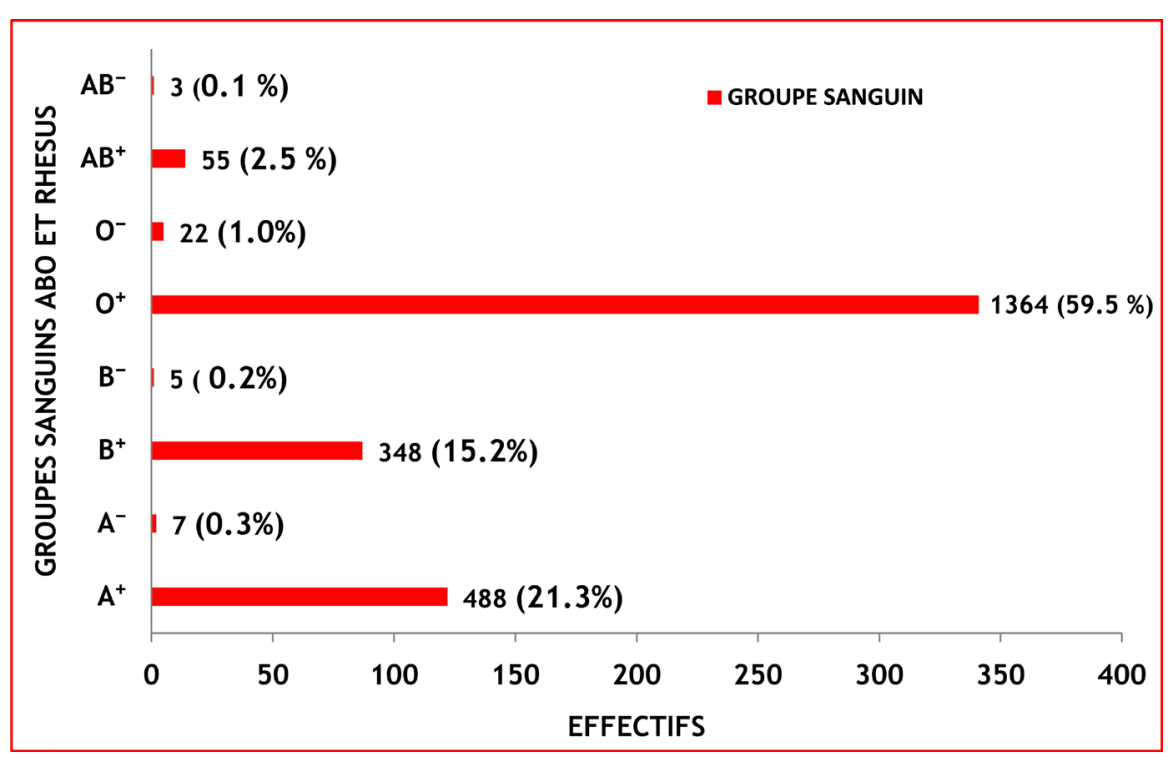

Figure 2. Distribution des Groupes sanguins ABO et Rhésus à Moba. Distribution of Abo and rhesus blood groups at Moba.

groupes sanguins $\mathrm{ABO}$ et Rhésus (D) comprenait par ordre d'importance: $\mathrm{O}^{+}$(n $=1364$ ou $59.5 \%) ; \mathrm{A}^{+} \mathrm{n}=488$ ou $\left.21.3 \%\right) ; \mathrm{B}^{+}(\mathrm{n}=348$ ou $15.2 \%) ; \mathrm{AB}^{+}(\mathrm{n}=55$ ou $2.4 \%) ; \mathrm{O}^{-}(\mathrm{n}=22$ ou $1.0 \%) ; \mathrm{A}^{-}(\mathrm{n}=7$ ou $0.3 \%) ; \mathrm{B}^{-}(\mathrm{n}=5$ ou $0.2 \%) ;$ et $\mathrm{AB}^{-}(\mathrm{n}=3$ ou $0.1 \%$ ) (Table 4 et Figure 2). Cette différence observée entre les différents groupes $\mathrm{ABO}$ et Rhésus est significative $(\mathrm{p}<0.05)$.

Les groupes sanguins $\mathrm{O}^{+}(\mathrm{n}=1364$ ou $59.5 \%)$ et $\mathrm{AB}^{-}(\mathrm{n}=3$ ou $0.1 \%)$ ont été respectivement le plus fréquent et le moins rencontré. Il est plus facile de rencontrer le rhésus $D$ négatif chez les femmes $(n=22)$ que pour les hommes $(n$ $=14$ ). Mais cette différence n'est pas statistiquement significative. 


\section{Discussion}

Nous avons étudiés la distribution des groupes sanguins $A B O$ et rhésus, dans le but de contribuer à la médecine transfusionnelle à Moba. L'Organisation Mondiale de la Santé (OMS) et le Programme Nationale de Transfusion Sanguine (PNTS) recommandent de transfusion du sang iso-groupe et iso-rhésus compatible pour la sécurité du receveur [29]. C’est dans ce cadre que notre étude trouve de l'intérêt.

Dans notre série d'étude sur les groupes sanguins $A B O$ et Rhésus chez les donneurs de sang de l'HGR de Moba, l'âge moyen des sujets inclus a été de 37.6 \pm 1.8 ans avec les extrêmes allant de 15 à 67 ans.

Notre travail révèle une légère prédominance non significative du sexe féminin parmi les individus sélectionnés pour cette étude. Il représente $50.4 \%$ contre $49.6 \%$ pour le sexe masculin (différence négligeable), ce qui est différent de certains auteurs [30] [31] [32] [33] pour qui ce sont des hommes qui constituent le groupe le plus important des donneurs de sang. Nous pensons que dans notre cas le hasard a plus joué, la culture exposant les hommes à se sentir plus fort et ainsi de tendre à plus donner le sang.

Il est tout de même de noter que l'OMS et les PNTS déconseillent aux individus de moins de 18 ans et plus de 65 ans d'être donneurs de sang [34], ce qui est contraire à notre constat sur terrain en utilisant les donneurs de sang comme échantillon pour cette étude. Il sied de souligner que ces personnes sont fragiles et nous devrions les éviter parmi les donneurs de sang, surtout dans nos zones où les cas de malnutrition sont plus rencontrés.

A Moba, le groupage sanguin est essentiellement demandé dans le cadre de compatibilité transfusionnelle, surtout pour les anémies décompensées dans le cadre de paludisme grave. La transfusion est un acte médical courant et salutaire qui permet de sauver nombreuses vies et réduire la morbi-mortalité, mais elle n'est pas anodine et est entachée de nombreux risque dont l'usage abusif pourrait exposer les receveurs à plusieurs complications d'ordre mécanique, infectieux et immunologique [29] [35]. Il était donc nécessaire pour nous de mener cette première étude pour avoir les données relatives à la répartition des groupes sanguins dans la population et ainsi améliorer la sécurité immunologique du receveur dans la gestion du sang à transfuser.

Le groupe sanguin est une classification du sang reposant sur la présence ou l'absence des antigènes hérités à la surface des cellules hématopoïétiques ou dans le plasma [7] [8] [9] [10]. La transmission des groupes sanguins suit les principes génétiques [26].

Les hommes se différencient génétiquement les uns des autres par leurs génotypes et phénotypes qui pourraient provenir de l'origine géographique au passé lointain puisque les fréquences de groupes sanguins varient d'un milieu à l'autre [8] [26] [36] [37].

En effet, la fréquence des antigènes $\mathrm{ABO}$ et Rhésus varie considérablement d'une population à l'autre, avec certaines influences liées discrètement aux brassages ethnique et social [26]. Les mariages hétéro-raciaux et hétéro- 
ethniques sont les preuves des moyens utilisés en faveur de cette influence.

Cette étude révèle qu'à Moba, le groupe $\mathrm{O}$ est plus représenté (60.5\%) suivi de A $(21,6 \%), B(15.4 \%)$ et $\mathrm{AB}(2.5 \%)$. Cette prédominance du groupe sanguin érythrocytaire $\mathrm{O}$ correspond aux données mondiales et dans quasiment tous les pays [4] [7] [15] [19] [22] [38] [39]. Nos résultats se rapprochent de ceux de Kalemie [7]: A (18.5\%), B (17.5\%), 0 (61.4\%) et AB (2.5\%). Omotade et al. [21] au Nigeria évoquait également en 1999 la primauté du groupe 0 (54.2\%) suivi de A (21.6\%), B (21.4\%) et AB (2.8\%); Falusi et al. [19] 70.7\% pour le O suivi de A (14.9\%), B (14.0\%) et AB (0.4\%); Lyko et al. [22] au Kenya 47.4\% de O, 26.2\% A, $22 \%$ B et $4.4 \%$ AB; Mwangi [20] 45\% de O, 27\% B, 23\% A et 5\% AB. Le groupe A a été le plus rencontré en Jordanie par Hanania et al. [26] avec 38.04\% pour A, $37.14 \% \mathrm{O}, 14.28 \%$ B et $9.52 \% \mathrm{AB}$. En Iran, Ghasemi et al. [11] avait noté la prédominance du groupe $\mathrm{B}$ avec $30.6 \%$ contre $30.6 \%$ pour le groupe $\mathrm{O}, \mathrm{AB} 27 \%$ $\mathrm{AB}$ et $12 \%$.

Pour le système du groupe sanguin rhésus, marqueur important en médecine transfusionnelle et obstétrique, notre travail ne fait pas exception de souligner comme tous les auteurs [3] [7] [12] [22] [38] [39] du monde entier la prédominance des rhésus positifs (98.4\%) alors que les rhésus négatifs ne représentent que $1.6 \%$. Lyko [22] avait évoqué $96.1 \%$ de rhésus (+) contre 3.9\% de Rhésus (-); Bakare et all. [36] 96.7\% de rhésus positifs; Eru et all. [6] 96.7\% Rh D positifs; Mwangi [20] 94\% de rhésus (+) pour les indigènes d'Afrique.

Dans cette étude, les groupes $O$ et $A B$ sont des groupes sanguins extrêmes. La littérature médicale consultée nous renseigne que c'est au Pakistan où la proportion des $\mathrm{AB}$ a été la plus grande avec 11.2\% [40].

L'ordre numérique de fréquence des phénotypes des groupes sanguins $A B O$ et Rhésus (D) comprend significativement de manière décroissante: $\mathrm{O}^{+}(\mathrm{n}=1364$ ou $59.5 \%) ; \mathrm{A}^{+} \mathrm{n}=488$ ou $\left.21.3 \%\right) ; \mathrm{B}^{+}(\mathrm{n}=348$ ou $15.2 \%) ; \mathrm{AB}^{+}(\mathrm{n}=55$ ou $2.4 \%) ;$ $\mathrm{O}^{-}(\mathrm{n}=22$ ou $1.0 \%) ; \mathrm{A}^{-}(\mathrm{n}=7$ ou $0.3 \%) ; \mathrm{B}^{-}(\mathrm{n}=5$ ou $0.2 \%) ;$ et $\mathrm{AB}^{-}(\mathrm{n}=3$ ou $0.1 \%)$. Il existe à ce point une similarité entre cette étude et celle de Kalemie [7]: $\mathrm{O}^{+}(60.6 \%), \mathrm{A}^{+}(17.5 \%), \mathrm{B}^{+}(17.0 \%), \mathrm{AB}^{+}(1.9 \%), \mathrm{O}^{-}(1.0 \%), \mathrm{A}^{-}(1.0 \%), \mathrm{AB}^{-}$ $(0.6 \%)$ et $\mathrm{B}^{-}(0.5 \%)$. Pour Idowu [4] au Nigeria $\mathrm{O}^{+}(49.75 \%), \mathrm{A}^{+}(20.30 \%), \mathrm{B}^{+}$ (19.60\%), $\mathrm{AB}^{+}(3.7 \%), \mathrm{O}^{-}(3.95 \%), \mathrm{B}^{-}(1.35 \%), \mathrm{A}^{-}(1.10 \%)$ et $\mathrm{AB}^{-}(0.25 \%)$. Imoru et al. [39] évoque $\mathrm{O}^{+}$(50.0\%), $\mathrm{A}^{+}(20.12 \%), \mathrm{B}^{+}(19.6 \%), \mathrm{AB}^{+}(2.8 \%), \mathrm{O}^{-}(2.3 \%)$, $\mathrm{A}^{-}(1.3 \%), \mathrm{B}^{-}(1.1 \%)$ et $\mathrm{AB}^{-}(0.4 \%)$. Les groupes $\mathrm{O}^{+}$et $\mathrm{A}^{+}$sont donc les plus rencontrés alors que $\mathrm{B}^{-}$et $\mathrm{AB}^{-}$les moins enregistrés ou rares.

Le Table 5 fait la comparaison de nos résultats en RDC et ceux des autres études dans le monde. Nous constatons que l'allure négroïde en Afrique avec une large prédominance du groupe $\mathrm{O}$ semble être respecté alors que les populations d'origine européennes et asiatiques ont respectivement une tendance générale à avoir les groupes A et B [7] [23] [36] [41] [42] [43].

\section{Conclusion}

La fréquence des groupes sanguins $\mathrm{ABO}$ et Rhésus est similaire que dans plusieurs pays avec une nette prédominance du groupe 0 et rhésus positif. Ces 
Table 5. Comparaison des résultats des études sur les phénotypes des groupes sanguins $\mathrm{ABO}$ et Rhésus Comparison of studies results about phenotypes of blood groups Abo and Rhesus.

\begin{tabular}{|c|c|c|c|c|c|c|c|c|}
\hline Auteurs ou Etudes & Pays & Année & GS A & GS B & GS O & GS AB & $\mathrm{RH}+$ & RH - \\
\hline CETTE ETUDE & $\mathrm{RDC}$ & 2017 & 21.6 & 15.4 & 60.5 & 205 & 98.4 & 1.6 \\
\hline IDOWU [4] & NIGERIA & 2008 & 21.4 & 21.0 & 52.7 & 4.0 & 93.3 & 6.7 \\
\hline SAID [44] & TUNISIE & 2003 & 37.6 & 22.5 & 59.3 & 6.7 & - & - \\
\hline INST [45] & France & 2015 & 45 & 9.1 & 43 & 2.9 & 84.9 & 15.1 \\
\hline LYKO [22] & KENYAN & 1992 & 26.2 & 22.0 & 47.4 & 4.4 & 96.1 & 3.9 \\
\hline DEWAN [43] & COLOMBIE & 2016 & 51.2 & 8.7 & 37 & 3.1 & 91.3 & 8.7 \\
\hline LOUA [42] & GUINEE & 2007 & 22.5 & 23.7 & 48.9 & 4.7 & 95.9 & 4.1 \\
\hline GARRATTY [46] & USA & 2004 & 37.1 & 12.2 & 46.6 & 4.1 & 85.4 & 14.6 \\
\hline CHANDRA [47] & INDE & 2012 & 21.7 & 39.8 & 29.1 & 9.3 & 95.7 & 4.3 \\
\hline KABEMBA [7] & $\mathrm{RDC}$ & 2016 & 18.5 & 17.5 & 61.6 & 2.5 & 97.0 & 3.0 \\
\hline BENAHADI [25] & MAROC & 2013 & 32.7 & 15.8 & 46.7 & 4.5 & 91 & 9 \\
\hline TORABIZADE [3] & IRAN & 2015 & 28.5 & 24.7 & 40.2 & 6.6 & 92.4 & 7.6 \\
\hline RANDRIAMANANTANY [48] & MADAGASCAR & 2012 & 22.6 & 29.7 & 41.6 & 6.1 & 98.9 & 1.1 \\
\hline NDOULA [24] & CAMEROUN & 2014 & 25.1 & 21.9 & 48.6 & 4.4 & 96.3 & 3.7 \\
\hline TESFAYE [49] & ETHIOPIE & 2015 & 28.1 & 23.1 & 43.1 & 5.4 & 92.1 & 7.9 \\
\hline KAYIRAN [41] & TURQUIE & 2012 & 38.2 & 14.4 & 29.7 & 6.8 & 89.6 & 10.4 \\
\hline MAINFRAY [18] & BRESIL & 1990 & 44 & 10 & 42 & 4 & - & - \\
\hline APECU [50] & OUGANDA & 2016 & 24.6 & 20.7 & 50.3 & 4.5 & 98.0 & 2.0 \\
\hline
\end{tabular}

résultat devraient être pris en considération pour planifier la collecte de sang à garder à la banque de sang dans de cadre de sécurité transfusionnelle: iso-groupe et iso-rhésus. Nous devrions être prudent sur les groupes sanguins rares (surtout RH négatif), d'où la nécessité de maitriser des adresses des donneurs potentiels.

\section{Conflit D'intérêt}

Nous ne déclarons aucun conflit d'intérêt.

\section{Financement}

Cette étude a été réalisée grâce aux contributions financières des auteurs.

\section{References}

[1] Bioforma (2000) Immuno-hématologie et groupes sanguins: Aspects théoriques, applications cliniques et transfusionnelles. Bioforma, Paris, No. 26, 31-53.

[2] Calot, M., Van Huffel, V. and Peyrard, T. (2002) Les bases théoriques des groupes sanguins Abo-Rh-Kell et phénotypes attendus et anticorps. INST, France, Février, $1-9$

[3] Torabizade Maatoghi, J., Paridar, M., Mahmodian Shoushtari, M., Kiani, B., Nori, B., Shahjahani, M., et al. (2016) Distribution of ABO Blood Groups and Rhesus Factor in a Large Scale Study of Different Cities and Ethnicities in Khuzestan Pro- 
vince, Iran. The Egyptian Journal of Medical Human Genetics, 17, 105-109. https://doi.org/10.1016/j.ejmhg.2015.07.004

[4] Oluwadare, I. and Shonekan, S. (2008) ABO and Rhesus "D" Blood Type Distribution in Students Admitted into Moshood Abiola Polytechnic, Abeokuta, Nigeria in 2006. African Journal of Biotechnology, 7, 1641-1643.

https://doi.org/10.5897/AJB08.216

[5] Acquaye, J.K. (2004) ABO Rhesus and Kell Groups in the Akans of Ghana. Ghana Medical Journal, 38, 68-71.

[6] Eru, E.U., Adeniyi, O.S. and Jogo, A.A. (2014) A-B-O and Rhesus Blood Group Distribution among Students of Benue State University Makurdi. Nigeria. African Journal of Biomedical Research, 17, 49-52.

[7] Kabemba Bukasa, H., Feza Malisuma, G., Mukena Tchioni, S., Shiku Muteba, A. and Ndjibu Muepu, J. (2016) Profil épidémiologique des groupes sanguins Abo et Rhésus à Kalemie (RD Congo): A propos de 1440 cas observes. Medecine d Afrique Noire, 63, 287-291.

[8] Onanuga, A. and Lamikanra, A. (2016) Association of ABO Blood Group and Plasmodium falciparum Malaria among Children in the Federal Capital Territory, Nigeria. African Journal of Biomedical Research, 19, 1-6.

[9] Sabir, B., Cherkaou, M., Baali, A., Hachri, H., Lamaire, O. and Dugoujon, J.M. (2004) Les dermatoglyphes digitaux et les groupes sanguins ABO, Rhésus et Kell dans une population Berbère de Haut-Atlas de Marrakech. Colloques du GALF Anthropo., 7, 211-221.

[10] Storry, J.R. and Olsson, M.L. (2009) The ABO Blood Group System Revisited: A Review and Update. Immunohematology, 25, 48-59.

[11] Ghasemi, N., Ayatollahi, J., Zadehrahmani, M., Nasiri, A., Abedi, A., Shokraneh, S. et al. (2010) Frequency of ABO and Rh Blood Groups in Middle School Students of Yazd Province. Iranian Journal of Pediatric Hematology and Oncology, 1, 27-30.

[12] Ikponmwen, D.O., Nwogoh, B. and Isoa, M.E. (2012) Distribution of ABO and Rhesus D Blood Groups among the Bini Ethnic Group of Southern Nigeria. Annals of Biomedical Sciences, 11, 2. www.ajol.info/index.php/abs/article/view/84428

[13] Johnson, P.H. and Hopkinson, D.A. (1992) Detection of ABO Blood Group Polymorphism by Denaturing Gradient Gel Electrophoresis. Human Molecular Genetics, 1, 341-344. https://doi.org/10.1093/hmg/1.5.341

[14] Bianco-Miotto, T., Hussey, D.J., Day, T.K., O'Keefe, D.S. and Dobrovic, A. (2009) DNA Methylation of the ABO Promoter Underlies Loss of ABO Allelic Expression in a Significant Proportion of Leukemic Patients. PLOS ONE, 4, e4788.

[15] Cartron, J.P. and Agre, P. (1993) Rh Blood Group Antigens: Protein and Gene Structure. Seminars in Hematology, 30, 193-208.

[16] Damulak, O.D., Bolorunduro, S., Deme, K.S., Yakubu, R.S., Zhakom, P.N. and Tokbam, L. (2011) ABO Blood Group Distribution among Voluntary Blood Donors in North Central Nigeria: The Implications on Blood Units Expiration. Journal of Medicine in the Tropics, 13, 2. www.ajol.info/index.php/jmt/article/view/70713 https://doi.org/10.4314/jmt.v13i2.70713

[17] Charlotte, P. (2010) Groupes sanguins dans le monde. Journal des femmes. www.sante.journaldesfemmes.com/magazine/groupes-sanguinsdu

[18] Mainfray, A. (1990) Recherche de corrélation entre groupes sanguins dans les systèmes ABO-Rhésus et pathologies au CHU Sao Polo, Brésil. Recherche en Soins Infirmiers, 20, 32.

[19] Falusi, A.G., Ademowo, O.G. and Latunji, L.A. (2000) Distribution of ABO and Rh Genes in Nigeria. African Journal of Medicine and Medical Sciences, 29, 23-26. 
[20] Mwangi, J. (1999) Blood Group Distribution in an Urban Population of Patient Targeted Blood Donors. East African Medical Journal, 76, 615-618.

[21] Omotade, O.O., Adeyemo, A.A., Kayode, C.M., Falade, S.L. and Ikpeme, S. (1999) Gene Frequencies of $\mathrm{ABO}$ and $\mathrm{Rh}(\mathrm{D})$ Blood Group Alleles in a Healthy Infant Population in Ibadan, Nigerian. West African Journal of Medicine, 18, 294-297.

[22] Lyko, J., Gaertner, H., Kaviti, J.N., Kariithi, M.W. and Akoto, B. (1992) BloodGroup Systems $\mathrm{ABO}$ and $\mathrm{Rh}$ in the Kenyan Population. Folia Medica Cracoviensia, 33, 85-92.

[23] Pughikumo, D.T., Onyebuagu, P.C. and Pughikumo, O.C. (2014) A Preliminary Study of Blood Groups among Students in Bayelsa State. International Journal of Basic Applied and Innovative Research, 23, 19-22.

[24] Ndoula, S.T., Noubiap, J.J.N., Nansseu, J.R.N. and Wonkam, A. (2014) Phenotypic and Allelic Distribution of the ABO and Rhesus (D) Blood Groups in the Cameroonian Population. International Journal of Immunogenetics, 41, 206-210. https://doi.org/10.1111/iji.12114

[25] Benahadi, A., Alami, R., Boulahdid, S., Adouani, B., Laouina, A., Mokhtari, A., et al. (2013) La distribution des antigenses de groups sanguins ABO et Rhesus D au Maroc. Transfusion clinique et biologique, 20, 313-317. https://doi.org/10.1016/j.tracli.2013.03.077

[26] Hanania Sahar, S., Hassawi Dhia, S. and Irshaid Nidal, M. (2007) Allele Frequency and Molecular Genotypes of ABO Blood Group System in a Jordanian Population. Journal of Medical Science, 7, 51-58. https://doi.org/10.3923/jms.2007.51.58

[27] Cellule d'analyse des indicateurs de développement (CAID)/RDC: Territoire de Moba.

www.caid.cd.index.php/donnees-par-province-administrative-province-de-tangany $\underline{\mathrm{ika} / \text { territoire-d-moba/?selecteur=fiche }}$

[28] Réseau national de laboratoires du Sénégal (RNL) (2004) Manuel de procédures des techniques de laboratoires d'analyses médicales, ministère de la santé et de la prévention médicale, République du Sénégal. RNL, Janv, 50-57.

[29] Kayiba, N., Tshibangu, E., Nsenda Nkongolo, J., Tshiunza, C. and Disashi, G. (2014) L'anémie palustre sévère à Mbuji-Mayi (RDC). Revue médicale des Grands Lacs, 3 , 78-103.

[30] Kra, O., N’Dri, N., Ehui, E., Ouattara, B. and Bissagnene, E. (2007) Prévalence de l'Antigène HBS chez les donneurs de sang et au centre régional de transfusion sanguine de Bouaké (Côte d'Ivoire) en 2001. Bulletin de la Société de Pathologie Exotique, 100, 127-129.

[31] Rakotoniaina, A.I., Randriamanantany, Z.A., Ranaivosoa, K.H.M, Andriambelo, V., Fortune, H., Rakoto Alson, O.A., et al. (2013) Séroprévalence du VIH, VHB, VHC et de Treponema pallidum chez les donneurs du sang bénévoles au centre national de transfusion sanguine d'Antananarivo de 1992 à 2010. La Revue Médicale de Madagascar, 3, 264-268.

[32] Dray, X., Dray-Spira, R., Bronstein, J.A. and Mattera, D. (2005) Séroprévalence des virus de l'immunodéficience humaine et des hépatites $\mathrm{B}$ et $\mathrm{C}$ parmi les donneurs de sang en République de Djibouti. Tropical Medicine, 65, 39-42.

[33] Kabinda, J.M., Miyanga, S.A., Misingi, P. and Ramarani, S.Y. (2014) Les hépatites B et $\mathrm{C}$ chez les donneurs bénévoles de sang et non rémunérés à l'Est de la République Démocratique du Cong. Transfusion Clinique et Biologique, 21, 111-115. https://doi.org/10.1016/j.tracli.2014.04.001

[34] OMS (2016) Que savez-vous du don de sang. Rubrique journée mondiale du donneur de sang. www.who.int 
[35] OMS (2015) Sécurité transfusionnelle et approvisionnement en sang, Aide-mémoire $\mathrm{n}^{\circ} 279$. www.who.int

[36] Bakare, A.A., Azeez, M.A. and Agbolade, J.O. (2006) Gene Frequencies of ABO Frequencies of $\mathrm{ABO}$ and Rhesus Blood Groups and Haemoglobin Variants in Ogbomoso, South-West Nigeria. African Journal of Biotechnology, 5, 224-229.

[37] Pramanik, T. and Pramanik, S. (2000) Distribution of ABO and Rh Blood Groups in Nepalese Students: A Report. Eastern Mediterranean Health Journal, 6, 156-158.

[38] Onoja, O.A., Ogli, S.A., Odutola, A.A. and Enyikwola, O. (2013) The Distribution of the $\mathrm{ABO}$ and Rhesus Blood Groups among an Indigenous Ethnic Group in Nigeria. Highland Medical Research Journal, 13, 81-84.

[39] Imoru, M., Onwuka, C., Kwaru, H. and Nwankwo, E. (2005) Distribution of ABO and Rhesus (D) Blood Groups in Kano Metropolis. Highland Medical Research Journal, 3, 139-143. www.ajol.info/index.php/hmrj/article/view/33884

[40] Khan, M.S., Farooq, N., Qamar, N., Tahir, F., Subhan, F., Kazi, B.M., et al. (2006) Trend of Blood Groups and Rh Factor in the Twin Cities of Rawalpindi and Islamabad. Journal of Pakistan Medical Association, 56, 299-302.

[41] Kayiran, S.M., Oktem, O., Kayiran, P.G., Paloglu, E. and Gurakan, B. (2012) Frequency of $\mathrm{ABO}$ and Rhesus Blood Groups among Neonates Born at a Private Hospital in Istanbul. The Southeast Asian Journal of Tropical Medicine and Public Health, 43, 467-470.

[42] Loua, A., Lamah, M.R., Haba, N.Y. and Camara, M. (2007) Frequency of Blood Groups $\mathrm{ABO}$ and Rhesus D in the Guinean Population. Transfusion Clinique et Biologique, 14, 435-439. https://doi.org/10.1016/j.tracli.2007.12.008

[43] Dewan, G. (2015) Comparative Frequency and Allelic Distribution of ABO and Rh (D) Blood Groups of Major Tribal Communities of Southern Bangladesh with General Population and Their Determinants. Egyptian Journal of Medical Human Genetics, 2, 141-147. https://doi.org/10.1016/j.ejmhg.2015.01.002

[44] Said, N., Ben Ahmed, F., Doghri, A., Ghazouani, E., Layouni, S., Gritli, N., et al. (2003) The ABO System Polymorphism in Tunisian Blood. Transfusion Clinique et Biologique, 10, 331-334. https://doi.org/10.1016/S1246-7820(03)00100-9

[45] INST (2015) Groupes sanguins. France. www.ints.fr/SangTransfGrSanguins.aspx

[46] Garratty, G., Glynn, S.A. and McEntire, R. (2004) ABO and Rh(D) Phenotype Frequencies of Different Racial/Ethnic Groups in the United States. Transfusion, 44, 703-706. https://doi.org/10.1111/j.1537-2995.2004.03338.x

[47] Chandra, T. and Gupta, A. (2012) Frequency of ABO and Rhesus Blood Groups in Blood Donors. Asian Journal of Transfusion Science, 6, 52-53.

https://doi.org/10.4103/0973-6247.95057

[48] Randriamanantany, Z.A., Rajaonatahina, D.H., Razafimanantsoa, F.E., Rasamindrakotroka, M.T., Andriamahenina, R., Rasoarilalamanarivo, F.B., et al. (2012) Phenotypic and Allelic Profile of ABO and Rhesus D Blood Group System among Blood Donor in Antananarivo. International Journal of Immunogenetics, 39, 477-479. https://doi.org/10.1111/j.1744-313X.2012.01120.x

[49] Tesfaye, K., Petros, Y. and Andargie, M. (2015) Frequency Distribution of ABO and $\mathrm{Rh}$ (D) Blood Group Alleles in Silte Zone, Ethiopia. Egyptian Journal of Medical Human Genetics, 16, 71-76.

[50] Apecu, R.O., Mulogo, E.M., Bagenda, F. and Byamungu, A. (2016) ABO and Rhesus (D) Blood Group Distribution among Blood Donors in Rural South-Western Uganda: A Retrospective Study. BMC Research Notes, 9, 513. https://doi.org/10.1186/s13104-016-2299-5 
Submit or recommend next manuscript to OALib Journal and we will provide best service for you:

- Publication frequency: Monthly

- 9 subject areas of science, technology and medicine

- Fair and rigorous peer-review system

- Fast publication process

- Article promotion in various social networking sites (LinkedIn, Facebook, Twitter, etc.)

- Maximum dissemination of your research work

Submit Your Paper Online: Click Here to Submit

Or Contact service@oalib.com 